\title{
Immunotherapy and targeted therapy-the new roadmap in cancer treatment
}

\author{
Kamran Shahid, Mustafa Khalife, Raetasha Dabney, Alexandria T. Phan \\ Division of Hematology \& Oncology, University of Texas Health Science Center at Tyler, UT Health North Campus Tyler, Tyler, TX, USA \\ Contributions: (I) Conception and design: K Shahid, AT Phan; (II) Administrative support: All authors; (III) Provision of study materials or patients: K \\ Shahid, AT Phan; (IV) Collection and assembly of data: K Shahid; (V) Data analysis and interpretation: K Shahid, AT Phan; (VI) Manuscript writing: \\ All authors; (VII) Final approval of manuscript: All authors. \\ Correspondence to: Alexandria T. Phan, MD. UT Health North Campus Tyler, University of Texas Health Science Center at Tyler, 11937 U.S. Hwy. \\ 271, Tyler, TX 75708, USA. Email: Alexandria.phan@uthct.edu.
}

\begin{abstract}
Immunotherapy is the new frontier in cancer medicine. This manuscript summarizes historical aspect of immunotherapy, particularly its pathway to drug approval as the main therapeutic modality used in clinical medicine. We will discuss the role of immunotherapy in treating various types of cancers and how the treatment landscape once dominated by chemotherapy is rapidly changing.
\end{abstract}

Keywords: Immunotherapy; chimeric antigen receptor T-cells (CAR T-cells); check point inhibitors; cancer treatment

Submitted May 07, 2019. Accepted for publication May 20, 2019.

doi: 10.21037/atm.2019.05.58

View this article at: http://dx.doi.org/10.21037/atm.2019.05.58

\section{Introduction}

Medicine has witnessed many discoveries in the last century. From the discovery of diphtheria anti-toxin leading to the first Nobel Prize in medicine awarded to Emil von Behring in 1901, to discovery of penicillin by Alexander Fleming, to treatment of HIV, there have been many breakthroughs over the years. This has been amply represented in the distribution of Nobel prizes in medicine and physiology over the years. Every era and every year depicting a breakthrough which has helped humanity in fighting ailments. If twentieth century was the antibiotics era, this century should be dubbed-the immunotherapy era, considering the difference it has already made in improving survival in cancer patients. In this context it would not be wrong to call the year 2018-the year of immunotherapy.

In 2018, the noble prize in medicine was awarded to basic science researcher, James Allison, who is rightfully dubbed as the father of check point inhibitors. Dr. Allison described the checkpoint molecule cytotoxic $\mathrm{T}$ lymphocyte associated protein 4 (CTLA-4) and its inhibitory function in the activation of T-cells. In mid 1990s, he showed anti-tumor effect in a series of mouse models by antibody blockade of CTLA-4 (1). This is the critical juncture in history of medicine which shifted the paradigm from attempting to activate the immune system that is vaccinating, to releasing the checkpoints that keep it in negative regulatory mode. This ultimately led to the development of check-point inhibitors and it was in 2011 when the U. S. Food and Drug Administration (FDA) approved ipilimumab. This was the first drug in this class which was noted to improve overall survival in metastatic melanoma (2).

\section{Immunotherapy versus chemotherapy}

Systemic therapy for cancer consists of anti-cancer agents administered into the system to damage or destroy cancer cells and hence cancer growth, which can be either molecularly targeted therapy, biological therapy such as immunotherapy or cytotoxic therapy. Cytotoxic therapy, also known as chemotherapy, has been traditionally thought of as the preferred weaponry for all cancers; it is now not a necessary therapeutic foundation for several malignancies. Many people compare giving cytotoxic systemic chemotherapy to "carpet bombing" in modern 
warfare where the idea is simple, the goal being to blast off foreign invasion called cancer, regardless of collateral damage. Compare this with check point inhibitors, a type of immunotherapy, very little of cytotoxic side-effects occur. However, check point blockade is associated with a unique spectrum of side effects called immune related adverse events (irAEs) (3). These usually occur due to hyperactivity of the immune system. Grade 1 and 2 irAEs are usually manageable by temporary interruption of treatment and the use of corticosteroids to suppress this hyperdrive of the immune system. Grade 3 and 4 irAEs rarely occurred with immunotherapy, but they are nevertheless managed by high dose of intravenous corticosteroids and other potent immunosuppressive agents like infliximab (4).

Since the first check point inhibitor ipilimumab, newer check point inhibitors are being developed and FDA approved, with much less severity and frequency of irAEs. The field of immunotherapy in cancer care has significantly expanded, with additional monoclonal antibodies differently directed against CTLA-4, programmed cell death protein 1 (PD-1) and programmed death-ligand 1 (PD-L1). Nivolumab is a human immunoglobulin G4 (IgG4) monoclonal antibody that binds to the $\mathrm{PD}-1$ receptor and blocks its interaction with PD-L1, releasing PD-1 pathway-mediated inhibition of the immune response, including the anti-cancer immune response. Results from the CheckMate 026 (5) and CheckMate 370 (6) clinical trials, comparing nivolumab with chemotherapy in patients with metastatic non-small cell lung cancer, showed revolutionary results of improved survival benefit. In 2015, nivolumab was initially FDA-approved for patients with advanced/metastatic non-small cell lung cancer and expanded to many other cancers since.

\section{Expanding impact of immunotherapy on current cancer care}

Since the first FDA-approval of ipilimumab treatment in solid malignancies had been significantly impacted by the introduction of immunotherapy particularly with immune checkpoint inhibitors. Immunotherapy was most transformative in the management and treatment of melanoma. Advanced or metastatic melanoma has always been considered the most virulent and resistant of all cancers, with dismal survival rates. The incidence of melanoma is currently on the rise with one-fifth of the diagnosed patients expected to develop metastatic disease (7). Treatment options were limited and included toxic chemotherapy and interferon treatments which had deleterious side-effects, including hypertriglyceridemia, hyperuricemia, hepatotoxicity, flu like symptoms, depressive mood disorder, suicidal thoughts among many others (8). One-year survival rate $25.5 \%$ and a 6.2 -month median survival duration (9). Ten-year survival rates improved to $22 \%$ with the monoclonal antibody directed against CTLA-4, compared to historical control of $10 \%(10,11)$. KEYNOTE-001 trial of pembrolizumab in patients with advanced/metastatic melanoma, demonstrated an improved 5 -year overall survival rate (OS) to $34 \%$ in all patients and $41 \%$ in treatment-naive patients (12). The study was one of the first to depict how much we have evolved in treating metastatic melanoma and with more tolerable irAEs. Table 1 summarizes for various immunotherapies used in clinical practice and the year they were approved for clinical use.

For advanced stage head and neck cancers, cytotoxic chemotherapy is still the first line treatment and prognosis are bad for patients who progress during treatment. Second line therapeutic options were limited, until the advent of introduction of immune checkpoint inhibitors. Nivolumab was the first immunotherapy FDA-approved in head and neck cancers because of the results from CheckMate-141 (13), followed by pembrolizumab FDAapproval for second-line therapy because of the results from KEYNOTE-40 (14).

In most cases, esophageal cancer is a treatable disease, but it is rarely curable in advanced or metastatic disease. Esophageal cancer is not as common in the U.S., but it has limited treatment options and prognosis is poor. Survival rates at 5 years for advanced stages esophageal cancer, is typically $5-20 \%$. Breakthrough in the search for effective second line treatment of patients with advanced esophageal cancers, came from findings of the KEYNOTE-181 trial. Results demonstrated pembrolizumab improve OS in patients with $\mathrm{PD}$ L1 combined positive score (CPS) $>10$ (15). CPS was developed to evaluate the number of PD-L1 staining cells relative to all viable tumor cells, and it has become a surrogate marker for patients who may benefit from treatment with pembrolizumab. The role of combination pembrolizumab and cytotoxic chemotherapy in esophageal cancers, is currently being studied in the frontline setting in an ongoing phase III trial, KEYNOTE-811 trial (16). In a phase II trial of patients with untreated metastatic gastric, gastroesophageal junctional and esophageal cancers overexpressing HER2NEU, the role of immune checkpoint inhibitor in combination with trastuzumab (monoclonal antibody against HER2NEU) has demonstrated preliminary 
Table 1 Selected Immunotherapy drugs in clinical practice

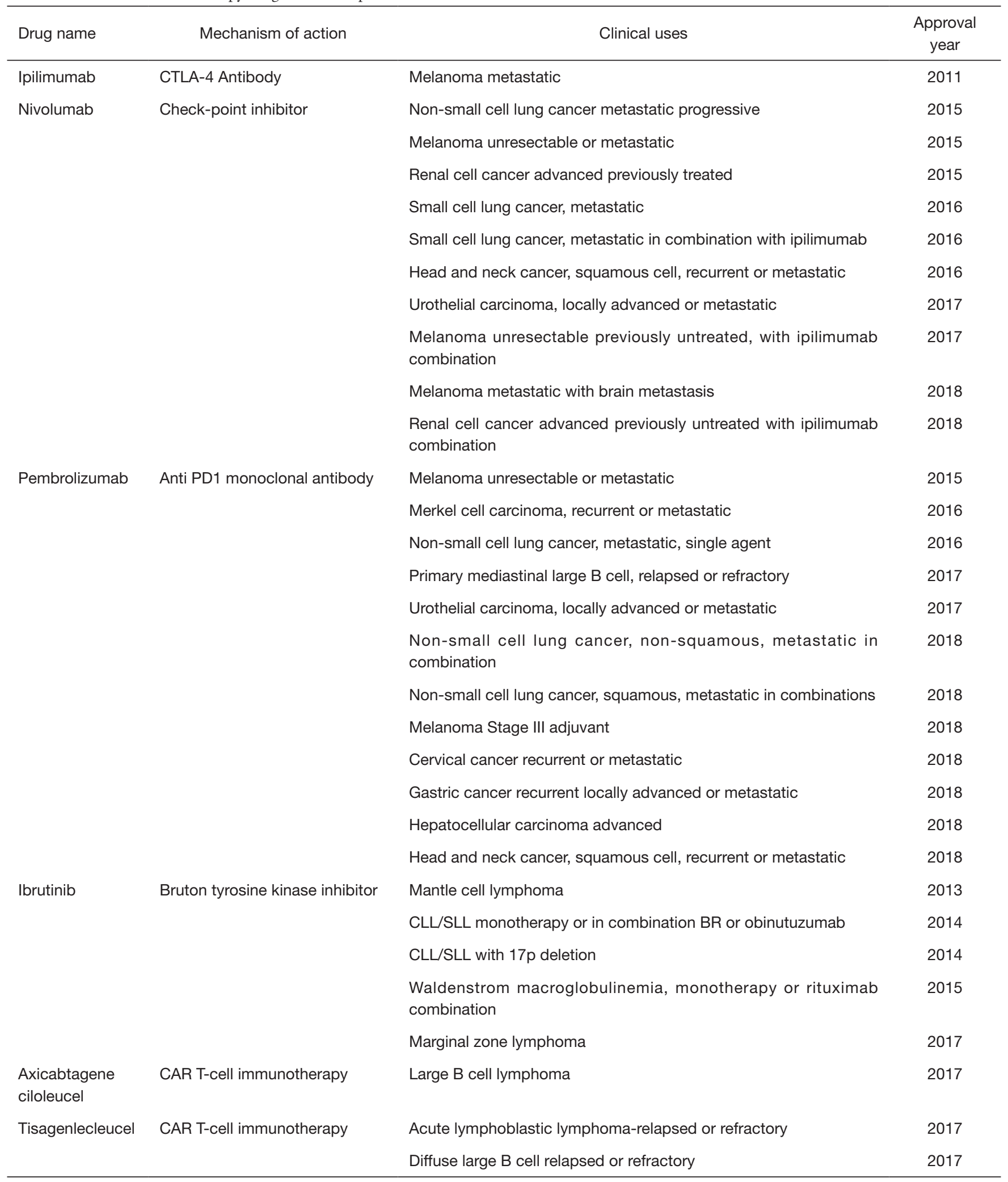


promising results, with median progression free survival reaching 11 months (17).

Dramatic improvement in survival benefits with immunotherapy compared to cytotoxic chemotherapy in lung cancers and melanoma, has led to the expanded development of immunotherapy in hematologic malignancies. In recent years, the paradigm for treatment of hematologic malignancies had dramatically changed. Gone are the days when fludarabine based combination chemotherapy was used to treat chronic lymphocytic leukemia (CLL) (18). Imagine the toxicity of chemotherapy compared to the newer treatment options in the form of immunotherapy. Ibrutinib is a small molecular drug that irreversibly binds to an important B cell enzyme, Bruton's tyrosine kinase (BTK). It is essentially the wonder drug being currently used to treat B-cell cancers like CLL, mantle cell lymphoma, and Wald Enstrom's macroglobulinemia, giving patients an effective chemotherapy-free option. Improved survival outcomes in both RESONATE 2 (19) comparing ibrutinib with chlorambucil and iLLUMINATE (20) comparing combination ibrutinib with obinutuzumab (fully humanized CD20 targeted monoclonal antibody) with standard chemoimmunotherapy regimen, validated current use of ibrutinib in front-line setting for patients with CLL. Additionally, the results of the iLLUMINATE trial continued to show progression free survival benefit even in high risk sub-groups (del17p or TP53 mutation, del11q or unmutated IGHV) compared with standard chemoimmunotherapy arm.

In classic Hodgkin lymphoma (cHL), investigators have evaluated the role of check point inhibitors to improve response rates (21-23). Pembrolizumab is another humanized IgG4 isotype antibody that binds to PD-1 located on lymphocytes and blocks the interaction of PD-L1 and PD-1. Results from KEYNOTE-087 (24) demonstrated that treating patients with relapsed refractory classic Hodgkin lymphoma with pembrolizumab, improved overall response rate. Similarly, treatment with combination of nivolumab and brentuximab vedotin, resulted in improved response rates for $\mathrm{cHL}$ patients in first relapse (25) and relapsing post-transplant (22). Based on these results and advances anti-PD-1 antibodies have now been FDAapproved for cHL patients with relapse and/or refractory diseases.

Immune checkpoint inhibitors are but one-way modulation of immune system infiltrate and revolutionize cancer care. Another success in immunotherapy is with
CAR (chimeric antigen receptor) T-cell therapy. CAR T-cell therapy is considered the most innovative example in personalized cancer medicine. This is a type of treatment in which a patient's own T-cells are changed in the laboratory so they will specifically attack that patient's cancer cells. These T-cells are taken from a patient's blood. Then the gene for a special receptor that binds to a certain protein on the patient's cancer cells is added in the laboratory, to genetically programmed and trained the T-cells to attack cancer cells. The special receptor is called a chimeric antigen receptor. Large numbers of the CAR T-cells are grown in the laboratory and infuse back into the patient. CAR T-cell therapy has been FDA-approved for certain types of lymphoma and leukemia since 2017. Results from ZUMA-1 (26) endorsed the FDA approval of axicabtagene ciloleucel (CD 19 directed CAR T-cell therapy) for patients with relapsed, refractory diffuse large B-cell lymphoma, follicular lymphoma with transformation and high-grade B-cell lymphoma with failure of two prior systemic lines of therapy. Tisagenlecleucel is another CD19-directed CAR T-cell therapy which was FDA-approved and currently being used for treatment of patients with B-cell precursor acute lymphoblastic leukemia that is refractory or in second or later relapse $(27,28)$. Complete response rates were $80-90 \%$ in this sub-group however the relapse free survival declined to 60 per cent in the 12 months and these are thought to be due to early CAR T-cell loss, also called T-cell exhaustion. Investigators are currently studying the use of immune checkpoint inhibitors in combination with CAR T-cell therapy to determine if the persistence of T-cell can be sustained, hence improving relapsed free survival.

\section{Future directions of immunotherapy and resistance mechanisms}

Immunotherapy with immune checkpoint inhibitors has replaced the foundation for many solid malignancies, notably melanoma and lung cancers. The impact is more dramatic in hematologic malignancies. Results from different clinical trials, have demonstrated the shift in treatment paradigm, where immunotherapy (both immune checkpoint inhibitors and CAR T-cell therapy) is completely changing the landscape which was previously occupied by cytotoxic chemotherapy or chemoimmunotherapy in hematologic malignancies. Are we looking at chemotherapy free future in all hematologic cancers? Not likely, as induction chemotherapy is still the standard of care in acute myeloid leukemia and aggressive non-Hodgkin lymphomas 
and clinical trials are ongoing to further evaluate the role of immunotherapy-based combination for these patients with aggressive and harder to treat hematologic malignancies. The search goes on for potential immunotherapy targets, resistance mechanisms and how to further enhance their responses. More and more cancer types which were previously considered non-immunogenic with limited role of immunotherapy are now showing signs of change. A very good example being triple negative, her 2 neu positive metastatic breast cancer where ongoing clinical trials show promising results (29).

Pancreatic cancer is another example which has been traditionally known to be resistant to immunotherapy. A detailed understanding of the cancer micro-environment now shows potential for VISTA pathway which can deactivate T-cells and hence suppress immune response leading to theoretical failure of PD-1/PDL1 inhibition. VISTA is expressed on macrophages which play a key role in innate immune response. Studying the milieu in pancreatic cancer shows increased macrophages in the stroma and much higher expression of VISTA pointing towards resistance mechanism for immunotherapy $(30,31)$. Other immune resistance pathway recently discovered is in K-ras mutant lung cancer. The MAPK signaling where expression of a protein called ZEB1 (presence of KRAS mutation is essential), targets the IL17RD protein suppression shutting down the pathway. This protein expression directs epithelial cells to transition to mesenchymal cell lines. This transition is important and noted in development and progression of various tumors (32). This also explains the reason why many tumors become resistant to treatment with the passage of time. This phenomenon called Epithelial to mesenchymal transition has been studied previously in dissemination of cancer cells (33).

Currently there are lot of molecular studies looking into immune resistant pathways and time will tell if we will be able to decipher them effectively to be clinically relevant. A more detailed understanding of the tumor microenvironment, role of small molecule Immuno-oncology, understanding resistance to NK cell immunotherapy against solid tumors, finding molecular markers of resistance, tumor evasion mechanisms and role of combining cytotoxic chemotherapy and its proper sequencing in treatment holds the key to the future.

\section{Conclusions}

Immunotherapy is currently becoming the new foundation stone in treatment of various malignancies and more recent clinical guidelines are constantly being updated to incorporate immunotherapy in the mix. This is a field which previously only included cytotoxic chemotherapy. Every researcher irrespective of the organ system is either studying it or trying to incorporate it into clinical trials. The next goal would be to determine sequencing of treatment options and which type of immunotherapy to choose from. CAR T-cells have shown promising results and a leap towards personalized medicine which without any doubts seem to be the future of oncology. Having achieved this status in cancer treatment starting from melanoma then lung cancer and now other cancers, we can only hope for better and better results in all cancer types including hematologic malignancies. New clinical trials are looking to enhance the excellent current clinical response rates achieved by immunotherapy and finding ways to overcome the known and unknown immune resistance pathways. With immunotherapy we are in the right direction and are looking forward to the day when cancer will just be called a chronic disease.

\section{Acknowledgments}

None.

\section{Footnote}

Conflicts of Interest: The authors have no conflicts of interest to declare.

\section{References}

1. Leach DR, Krummel MF, Allison JP. Enhancement of antitumor immunity by CTLA-4 blockade. Science 1996;271:1734-6.

2. Hersh EM, O'Day SJ, Powderly J, et al. A phase II multicenter study of ipilimumab with or without dacarbazine in chemotherapy-naive patients with advanced melanoma. Invest New Drugs;29:489-98.

3. YERVOY. Insert package of Ipilimumab 2016, August 10. Available online: http://packageinserts.bms.com/pi/pi_ yervoy.pdf

4. Howell M, Lee R, Bowyer S, et al. Optimal management of immune-related toxicities associated with checkpoint inhibitors in lung cancer. Lung Cancer 2015;88:117-23.

5. An open-label, randomized, phase 3 trial of nivolumab versus investigator's choice chemotherapy as first-line 
therapy for stage IV or recurrent PD-L1+ non-small cell lung cancer (CheckMate 026). National Library of Medicine (US), 2018.

6. A master protocol study of nivolumab in advanced nonsmall cell lung cancer (NSCLC) (CheckMate 370). National Library of Medicine (US), 2018.

7. Corrie P, Hategan M, Fife K, et al. Management of melanoma. Br Med Bull 2014;111:149-62.

8. Sleijfer S, Bannink M, Van Gool AR, et al. Side effects of interferon-alpha therapy. Pharm World Sci 2005;27:423-31.

9. Fava P, Astrua C, Sanlorenzo M, et al. Treatment of metastatic melanoma: a multidisciplinary approach. G Ital Dermatol Venereol;152:241-61.

10. Hodi FS, O'Day SJ, McDermott DF, et al. Improved survival with ipilimumab in patients with metastatic melanoma. N Engl J Med;363:711-23.

11. Schadendorf D, Hodi FS, Robert C, et al. Pooled Analysis of Long-Term Survival Data From Phase II and Phase III Trials of Ipilimumab in Unresectable or Metastatic Melanoma. J Clin Oncol 2015;33:1889-94.

12. Hamid O, Robert C, Daud A, et al. Five-year survival outcomes for patients with advanced melanoma treated with pembrolizumab in KEYNOTE-001. Ann Oncol. 2019;30:582-88.

13. Karabajakian A, Reverdy T, Gau M, et al. Nivolumab in recurrent/metastatic head and neck cancers. Future Oncol 2018;14:603-9.

14. Cohen EEW, Soulieres D, Le Tourneau C, et al. Pembrolizumab versus methotrexate, docetaxel, or cetuximab for recurrent or metastatic head-and-neck squamous cell carcinoma (KEYNOTE-040): a randomised, open-label, phase 3 study. Lancet 2019;393:156-67.

15. Shah MA, Kojima T, Hochhauser D, et al. Efficacy and Safety of Pembrolizumab for Heavily Pretreated Patients With Advanced, Metastatic Adenocarcinoma or Squamous Cell Carcinoma of the Esophagus: The Phase 2 KEYNOTE-180 Study. JAMA Oncol 2019;5:546-50.

16. Pembrolizumab/Placebo Plus Trastuzumab Plus Chemotherapy in Human Epidermal Growth Factor Receptor 2 Positive (HER2+) Advanced Gastric or Gastroesophageal Junction (GEJ) Adenocarcinoma (MK-3475-811/KEYNOTE-811). National Library of Medicine, 2019.

17. Tabernero J, Hoff PM, Shen L, et al. Pertuzumab plus trastuzumab and chemotherapy for HER2-positive metastatic gastric or gastro-oesophageal junction cancer (JACOB): final analysis of a double-blind, randomised, placebo-controlled phase 3 study. Lancet Oncol 2018;19:1372-84.

18. Hallek M, Fischer K, Fingerle-Rowson G, et al. Addition of rituximab to fludarabine and cyclophosphamide in patients with chronic lymphocytic leukaemia: a randomised, open-label, phase 3 trial. Lancet 2010;376:1164-74.

19. Burger JA, Tedeschi A, Barr PM, et al. Ibrutinib as Initial Therapy for Patients with Chronic Lymphocytic Leukemia. N Engl J Med 2015;373:2425-37.

20. Moreno C, Greil R, Demirkan F, et al. Ibrutinib plus obinutuzumab versus chlorambucil plus obinutuzumab in first-line treatment of chronic lymphocytic leukaemia (iLLUMINATE): a multicentre, randomised, open-label, phase 3 trial. Lancet Oncol 2019;20:43-56.

21. Ansell SM. Immunotherapy of Hodgkin Lymphoma: Mobilizing the Patient's Immune Response. Cancer J 2018;24:249-53.

22. Armand P, Engert A, Younes A, et al. Nivolumab for Relapsed/Refractory Classic Hodgkin Lymphoma After Failure of Autologous Hematopoietic Cell Transplantation: Extended Follow-Up of the Multicohort Single-Arm Phase II CheckMate 205 Trial. J Clin Oncol 2018;36:1428-39.

23. Foran AE, Nadel HR, Lee AF, et al. Nivolumab in the Treatment of Refractory Pediatric Hodgkin Lymphoma. J Pediatr Hematol Oncol 2017;39:e263-e6.

24. Chen R, Zinzani PL, Fanale MA, et al. Phase II Study of the Efficacy and Safety of Pembrolizumab for Relapsed/ Refractory Classic Hodgkin Lymphoma. J Clin Oncol 2017;35:2125-32.

25. Wang Y, Nowakowski GS, Wang ML, et al. Advances in CD30- and PD-1-targeted therapies for classical Hodgkin lymphoma. J Hematol Oncol 2018;11:57.

26. Neelapu SS, Locke FL, Bartlett NL, et al. Axicabtagene Ciloleucel CAR T-Cell Therapy in Refractory Large B-Cell Lymphoma. N Engl J Med 2017;377:2531-44.

27. KYMRIAH ${ }^{\mathrm{TM}}$ (tisagenlecleucel). FDA. 2018, May. Available online: https://www.fda.gov/downloads/ BiologicsBloodVaccines/CellularGene'TherapyProducts/ ApprovedProducts/UCM573941.pdf

28. Park JH, Riviere I, Gonen M, et al. Long-Term Followup of CD19 CAR Therapy in Acute Lymphoblastic Leukemia. N Engl J Med 2018;378:449-59.

29. Tolba MF, Omar HA. Immunotherapy, an evolving approach for the management of triple negative breast cancer: Converting non-responders to responders. Crit Rev Oncol Hematol 2018;122:202-7.

30. Blando J, Sharma A, Higa MG, et al. Comparison of 
immune infiltrates in melanoma and pancreatic cancer highlights VISTA as a potential target in pancreatic cancer. Proc Natl Acad Sci U S A 2019;116:1692-7.

31. Nowak EC, Lines JL, Varn FS, et al. Immunoregulatory functions of VISTA. Immunol Rev 2017;276:66-79.

32. Peng DH, Kundu ST, Fradette JJ, et al. ZEB1 suppression sensitizes KRAS mutant cancers to MEK inhibition by an IL17RD-dependent mechanism. Sci Transl Med 2019. doi: 10.1126/scitranslmed.aaq1238

33. Wu Y, Yang X, Chen Z, et al. m6A-induced lncRNA RP11 triggers the dissemination of colorectal cancer cells via upregulation of Zeb1. Mol Cancer 2019;18:87.

Cite this article as: Shahid K, Khalife M, Dabney R, Phan AT. Immunotherapy and targeted therapy-the new roadmap in cancer treatment. Ann Transl Med 2019;7(20):595. doi: 10.21037/ atm.2019.05.58 